\title{
Feeding Outcomes for Infants with Bronchopulmonary Dysplasia Discharged on Nasogastric Feeds
}

Preet Matharu ${ }^{1}$, A. loana Cristea ${ }^{1}$, James E. Slaven², Samantha Becker ${ }^{1}$, Jason Z. Niehaus $^{1}$

1. Department of Pediatrics, Riley Hospital for Children, Indiana University, Indianapolis, Indiana.

2. Department of Biostatistics, Indiana University School of Medicine, Indianapolis, Indiana.

Correspondence: Jason Z. Niehaus, MD. Division of Neonatal-Perinatal Medicine, Department of Pediatrics, Riley Hospital for Children, Indiana University. 1030 West Michigan Street, Suite C 4600, Indianapolis, IN 46202. Email: zzniehau@iu.edu

\begin{abstract}
Objective While previous studies regarding neonatal home feeding regimens have demonstrated a more favorable prognosis for nasogastric (NG) versus gastrostomy tubes (GT), institutional practices of discharge on NG feeds are varied, particularly for infants with bronchopulmonary dysplasia (BPD). Little is known regarding the risk factors for patients treated with GT postdischarge in premature infants with BPD. Our objective was to identify frequency and risk factors for treating premature infants with BPD discharged on NG feeds with GT.
\end{abstract}

Study Design In this retrospective study, we included infants born at 30 weeks' gestational age with BPD transitioning from a tertiary care center to home on NG feeds from 2010 to 2016.

Results Of the 86 infants included in this study, 25 (29\%) underwent GT placement at a median age of 264 days postdischarge. Fourteen (56\%) were able to remove the GT at a median age of 979.5 days. Infants not requiring GT placement postdischarge were found to have a significantly higher ccipitofrontal circumference $(p=0.0089)$ and length $(p=0.0166)$ at discharge compared with infants with GT.

Conclusion NG feeding for infants with BPD appears to be a viable treatment with fewer patients (29\%) requiring GT placement. Gestational age and abnormal magnetic 
resonance imaging results were found to have a significant association with GT placement postdischarge.

Keywords: Nutrition - Bronchopulmonary Dysplasia - Gastrostomy Tube - Premature 
For preterm infants, one of the criteria for discharge is to reach per os (PO) intake sufficient for growth prior to leaving the hospital. However, there is institutional variability in clinical practice when an infant is ready for discharge prior to maintaining adequate oral intake. For example, some infants are discharged on nasogastric (NG) feeds, allowing the child to continue to work on oral feeding skills outside of the hospital. Other institutions keep these infants inpatient until they can take enough nutrition PO to maintain appropriate growth and, when progress with oral feeding skills is inadequate, receive gastrostomy tubes (GT) and thereby avoid home NG feeding.

Several studies have compared the outcomes of neonates based on different feeding regimens.[1] [2] [3] It has been shown that more infants on NG feeds have demonstrated the ability to achieve full (PO) feeds by 6 months, with fewer emergency visits postdischarge compared with infants on GT.[1] Pediatric patients discharged on NG feeds have also been found to display significant improvement in measured growth parameters compared with those with GT.[2] [3] However, included in these studies were patients with various diagnoses and reasons for oral feeding difficulty, which makes extrapolation to a specific population difficult.

Multiple factors including hypotonia and bronchopulmonary dysplasia (BPD) have been described as major influences in determining the prerequisites for infants to have poor oral feeding skills.[3] [4] [5] [6] Regarding BPD, there is little information detailing specific feeding outcomes in premature infants postdischarge. In particular, there is insufficient data regarding the eventual need for GT and the types of clinical factors that may be associated with GT placement in infants with BPD. This lack of evidence makes it difficult to counsel families which clinical care path is best for their family and to establish appropriate expectations. We therefore conducted this study to determine long-term feeding outcomes of premature infants with BPD who are discharged home on NG feeds. Our secondary aim was to evaluate prenatal and postnatal factors associated with the eventual need for postdischarge GT placement. 


\section{MATERIALS AND METHODS}

We conducted a retrospective study involving infants discharged from an academic tertiary care center between January 2010 and December 2016. Infants with BPD were born at 30 weeks' gestational age or less and discharged home on NG feeds. For inclusion, BPD was diagnosed based on oxygen requirement at 28 days of life.[7] [8] These infants were assigned to one of two groups: those who eventually received GT postdischarge and those who did not. The primary outcome was to determine the number of GT placement procedures performed in infants meeting the inclusion criteria. Secondary outcomes were defined as the time to GT placement, whether the patients had successful GT removal, and time to removal after discharge. Clinical characteristics and demographics were compared between the two groups to determine factors associated with GT placement. For analysis, we further stratified severity of BPD based on respiratory support at 36 weeks adjusted age.[9] Abnormal magnetic resonance imaging (MRI) results were defined as any abnormality not consistent with gestational age at the time of imaging. Examples of abnormal results included but were not limited to intraventricular hemorrhage, hydrocephalus, cerebral atrophy, and hypoxic ischemic injury.

REDCap (Research Electronic Data Capture) was used for study data collection and management.[10] [11] Statistical analyses were performed with chi-square tests (Fisher's exact when cell counts were small) for categorical variables and Student's $t$-tests for continuous variables, with Wilcoxon's nonparametric tests being used when data were nonlinear. Results were presented as means (standard deviations) for linear continuous variables, medians (ranges) for nonlinear continuous variables, and frequencies (percentages) for categorical variables. Multivariable models were also performed for both demographic and clinical characteristics, with the inclusion of any variable that was significant at $p<0.20$ in the bivariate analyses. All analytic assumptions were verified and analyses were performed using SAS v9.4 (SAS Institute, Cary, NC). 


\section{RESULTS}

A total of 86 patients met the inclusion criteria. The demographics and clinical characteristics assessed for the patient population are illustrated in [Tables 1] and [2], respectively. The population was divided and analyzed according to whether or not the patient underwent postdischarge GT placement. There was a statistically significant difference in gestational age for patients treated with GT compared with those without GT ( 26.00 vs. 27.43 weeks, $p=0.02$ ). Our primary outcome was to determine the number of patients discharged on NG feeds requiring GT placement. Of the 86 infants, 29\% $(n=25)$ had a GT placed with median time to placement being 137 days after discharge. The median age at GT placement was 264 days. Of these patients, $56 \%(n=14)$ successfully underwent GT removal with median age at removal being 979.5 days.

Secondary outcomes of the study demonstrated that more infants with GT were found to have abnormal brain MRI results $(p=0.0446)$ compared with those without GT. Infants who did not require GT placement postdischarge had a significantly higher occipitofrontal circumference (OFC) $(p=0.01)$ and length $(p=0.02)$ at discharge compared with infants requiring GT. There were no significant differences between the groups when comparing based on the severity of BPD based on respiratory support at 36 weeks adjusted age. In both multivariable models, all results were attenuated, although effect sizes remained similar.

\section{DISCUSSION}

To address the fact that there is insufficient data regarding specific feeding outcomes in premature infants with BPD, this retrospective chart review study was conducted to establish new data regarding postdischarge GT placement rates of premature infants discharged home on NG feeds. In our cohort, a large majority of infants were able to successfully transition to PO feeds, while $29 \%$ required a GT postdischarge. Additionally, infants discharged on NG feeds who did not require a GT demonstrated more optimal growth (specifically in length and OFC) during their admission compared with infants who received a GT. This suggests that infants who have more optimal growth during their hospital admission are more likely to avoid a postdischarge GT. 
Our results reveal that younger birth gestational ages are more commonly associated with GT placement, a finding similar to other studies.[4] Positive growth outcomes seen in this study have also been reported in other studies regarding infants on NG feeds.[2] Comparable to previous studies, we identified that abnormal brain MRI results were also associated with poorer feeding outcomes requiring the use of GT.[12] However, the rate of GT placement in our study is different, with one study reporting GT placement of 10.3\%.[2] One potential explanation for this difference is the fact that we included only premature infants with BPD, thereby narrowing the inclusion criteria, whereas previous studies have also included nonpremature infants with other comorbidities.

In addition to postdischarge GT placements rates and traits, we also describe characteristics of infants able to have GT removed due to improved oral feeding skills. With $56 \%$ of placed GT able to be removed at a median age of 979.5 days, we will be able to provide a more tangible set of expectations for families and providers. This will help guide provider counseling and caregiver decision making as it pertains to postdischarge feeding natural history.

While we successfully provide data regarding GT placement in premature infants with BPD, there are nonetheless study limitations to consider. The retrospective nature of the study design and dependence on reliable medical records leaves room for reporting error in data collection. Additionally, it is possible that some potential subjects meeting the inclusion criteria were not included in the study due to loss of follow-up or inability to maintain records when transferring between institutions. The group of patients included in this study was also limited to a single tertiary care center which resulted in fewer subjects meeting the inclusion criteria. Practice variability between centers places limitations on generalizations regarding outcomes at institutions with very different discharge criteria.

With the data presented in our report, additional information is now available regarding the need for postdischarge GT placement in premature infants with BPD discharged home on NG feeds. This is a more homogenous population in regard to the etiology feeding dysfunction than previous studies. This information will facilitate health care providers and primary caregivers to make relevant decisions, as well as allow for 
appropriate expectations, regarding home-assisted feeding regimens for premature infants with BPD.

\section{REFERENCES}

1. Khalil ST, Uhing MR, Duesing L, Visotcky A, Tarima S, Nghiem-Rao TH. Outcomes of infants with home tube feeding: comparing nasogastric vs gastrostomy tubes. J Parenter Enteral Nutr 2017; 41 (08) 1380-1385

2. Rosen D, Schneider R, Bao R. , et al. Home nasogastric feeds: feeding status and growth outcomes in a pediatric population. J Parenter Enteral Nutr 2016; 40 (03) 350-354

3. Puia-Dumitrescu M, Benjamin Sr DK, Smith PB., et al. Impact of gastrostomy tube placement on short-term weight gain in hospitalized premature infants. J Parenter Enteral Nutr 2019 . Doi: 10.1002/jpen.1539

4. White BR, Zhang C, Presson AP, Friddle K, DiGeronimo R. Prevalence and outcomes for assisted home feeding in medically complex neonates. J Pediatr Surg 2019; 54 (03) 465470

5. Lee JH, Chang YS, Yoo HS. , et al. Swallowing dysfunction in very low birth weight infants with oral feeding desaturation. World J Pediatr 2011; 7 (04) 337-343

6. Crapnell TL, Rogers CE, Neil JJ, Inder TE, Woodward LJ, Pineda RG. Factors associated with feeding difficulties in the very preterm infant. Acta Paediatr 2013; 102 (12) e539e545

7. Tooley WH. Epidemiology of bronchopulmonary dysplasia. J Pediatr 1979; 95 (5 Pt 2): 851-858

8. Kair LR, Leonard DT, Anderson JM. Bronchopulmonary dysplasia. Pediatr Rev 2012; 33 (06) 255-263

9. Higgins RD, Jobe AH, Koso-Thomas M. , et al. Bronchopulmonary dysplasia: executive summary of a workshop. J Pediatr 2018; 197: 300-308

10. Harris PA, Taylor R, Thielke R, Payne J, Gonzalez N, Conde JG. Research Electronic Data Capture (REDCap)--a metadata-driven methodology and workflow process for providing translational research informatics support. J Biomed Inform 2009; 42 (02) 377381 
11. Harris PA, Taylor R, Minor BL. , et al; REDCap Consortium. The REDCap consortium: building an international community of software platform partners. J Biomed Inform 2019; 95: 103208

12. Limperopoulos C, Bassan H, Gauvreau K. , et al. Does cerebellar injury in premature infants contribute to the high prevalence of long-term cognitive, learning, and behavioral disability in survivors?. Pediatrics 2007; 120 (03) 584-593

Table 1

Demographic data of infants discharged to home on NG feeds

\begin{tabular}{|c|c|c|c|c|c|}
\hline Characteristic & All & G-tube, no & G-tube, yes & $\begin{array}{l}p- \\
\text { Value }\end{array}$ & $\begin{array}{l}\text { Odds } \\
\text { ratio }\end{array}$ \\
\hline Number[a] & 86 & $61(70.9)$ & $25(29.1)$ & NA & \\
\hline Female[a] $]$ & $41(47.7)$ & $27(44.3)$ & $14(56.0)$ & 0.3224 & 1.60 \\
\hline White[a] & $57(66.3)$ & $39(63.9)$ & $18(72.0)$ & 0.4725 & 1.45 \\
\hline $\begin{array}{l}\text { Gestational age } \\
(\mathrm{wk})[\underline{\mathrm{b}}]\end{array}$ & $\begin{array}{l}26.9(25.4, \\
28.0)\end{array}$ & $\begin{array}{l}27.4(26.1, \\
28.9)\end{array}$ & $\begin{array}{l}26.0(25.1, \\
27.3)\end{array}$ & 0.0207 & 0.52 \\
\hline$\leq 25$ wk[a] & $19(22.1)$ & $13(21.3)$ & $6(24.0)$ & 0.7849 & 0.86 \\
\hline$>25$ wk[a] & 67 (77.9) & $48(78.7)$ & $19(76.0)$ & - & \\
\hline Discharge age (wk)[b] & $\begin{array}{l}14.8(12.6, \\
17.1)\end{array}$ & $\begin{array}{l}14.0(11.7, \\
17.0)\end{array}$ & $\begin{array}{l}15.1(14.4, \\
17.6)\end{array}$ & 0.1111 & 0.34 \\
\hline Discharge weight, $z$ & $-0.16(1.01)$ & $-0.05(0.99)$ & $-0.42(1.03)$ & 0.1152 & 0.38 \\
\hline Discharge OFC, $z$ & $0.25(0.97)$ & $0.42(0.96)$ & $-0.17(0.87)$ & 0.0089 & 0.64 \\
\hline
\end{tabular}




\begin{tabular}{|l|l|l|l|l|l|}
\hline Discharge length, $z$ & $-0.88(1.32)$ & $-0.67(1.26)$ & $-1.41(1.35)$ & $\mathbf{0 . 0 1 6 6}$ & 0.58 \\
\hline Birth weight, $z$ & $0.08(0.98)$ & $0.17(0.94)$ & $-0.13(1.06)$ & 0.1879 & 0.32 \\
\hline Birth OFC, $z$ & $-0.05(1.12)$ & $0.06(1.17)$ & $-0.32(0.970$ & 0.1539 & 0.34 \\
\hline Birth length, $z$ & $-0.13(1.12)$ & $-0.03(1.10)$ & $-0.38(1.15)$ & 0.1945 & 0.31 \\
\hline
\end{tabular}

Abbreviations: NG, nasogastric; OFC, occipitofrontal circumference.

Note: All data presented as mean (standard deviation) unless otherwise noted.

a Mean (interquartile range $25 \%, 75 \%$ ).

${ }^{b} n(\%)$.

Table 2

Clinical characteristics of infants discharged to home on NG feeds

\begin{tabular}{|l|l|l|l|l|l|}
\hline Characteristic & All & G-tube, no & G-tube, yes & p-Value & Odds ratio \\
\hline Number, $n$ (\%) & 86 & $61(70.9)$ & $26(29.1)$ & NA & \\
\hline Intubated 36 wk & $9(10.5)$ & $6(9.8)$ & $3(12.0)$ & 0.7154 & 1.25 \\
\hline Periventricular leukomalacia & $5(5.8)$ & $3(4.9)$ & $2(8.0)$ & 0.5792 & 1.68 \\
\hline Intraventricular hemorrhage & $12(14.0)$ & $7(11.5)$ & $5(20.0)$ & 0.3002 & 1.93 \\
\hline Abnormal MRI & $19(22.1)$ & $10(16.4)$ & $9(36.0)$ & $\mathbf{0 . 0 4 4 6}$ & 2.87 \\
\hline Seizures & $6(7.0)$ & $2(3.3)$ & $4(16.0)$ & 0.0565 & 5.62 \\
\hline Surgical intervention & $8(9.3)$ & $6(9.8)$ & $2(8.0)$ & 1.0000 & 0.80 \\
\hline
\end{tabular}


Table 2

Clinical characteristics of infants discharged to home on NG feeds

\begin{tabular}{|c|c|c|c|c|c|}
\hline Characteristic & All & G-tube, no & G-tube, yes & p-Value & Odds ratio \\
\hline \multicolumn{6}{|l|}{ BPD category } \\
\hline Low $(0-1)$ & $54(62.8)$ & $38(70.4)[a]$ & 16 (29.6)[a] & 0.8819 & 0.93 \\
\hline $\operatorname{High}(2-3)$ & $32(37.2)$ & $23(71.9)[a]$ & $9(28.1)[a]$ & & \\
\hline
\end{tabular}

